\title{
Orbital magnetic phase and pure persistent spin current in spin-orbit coupling mesoscopic rings
}

\author{
GuAng-YaO HuAng and SHI-DONG LiAnG ${ }^{\dagger}$ \\ State Key Laboratory of Optoelectronic Material and Technology and \\ School of Physics and Engineering, Sun Yat-Sen University, Guangzhou, 510275, People's \\ Republic of China \\ † Corresponding author, Email: stslsd@mail.sysu.edu.cn
}

PACS 72.10.Bg - General formulation of transport theory

PACS 72.25.Dc - Spin polarized transport in semiconductors

\begin{abstract}
By solving the Rashba model of mesoscopic rings, we give analytically the ground-state properties of the ring, including the spin polarization, the persistent charge and spin currents (PCC and PSC). These ground-state properties can be given based on four kinds of electron numbers in rings. The effect of the self-inductance of the ring leads to the self-sustained magnetic flux (SSMF) and the self-sustained PCC and PSC, which break spontaneously time reversal symmetry to form orbital magnetic phase (OMP). To tune the spin-orbit coupling strength or electron number of the ring can induce the phase transition between the OMP and non-OMP. For exact one-dimensional rings we find the coexistence of the pure PSC and SSMF. This property of the pure PSC may provide a new scheme to measure the pure PSC.
\end{abstract}

Spintronics as a new scheme of quantum electronic devices has attracted much attention, whose central idea is to use the interplay between spin-orbit (SO) coupling and quantum confinement in semiconductor heterostructures. [1 3] The structural inversion asymmetry, namely Rashba effect, leads to intrinsic spin splitting in semiconductor heterostructures. 445] Many proposals have been put forward for devices based on spin-dependent transport effect due to the Rashba SO coupling in low-dimensional systems. 6] On the other hand, some quantum phase and quantum interference effects in low-dimensional systems have attracted great interests, 7] such as the Aharonov-Bohm (AB) oscillation of the ring conductance induced by the SO coupling, [8] and the persistent charge and spin currents (PCC and PSC) in semiconductor Rashba rings. 9 11] Although the PCC has been observed experimentally both in an ensemble of metallic rings [12] and single isolated rings [13, the experimental investigation of PCC and PSC in SO coupling semiconductor rings is still a challenging issue for experimental physicists. Interestingly, the pure PSC was predicted in SO coupling semiconductor rings, 11 and this pure PSC can generate an electric field, which provides a scheme to measure the pure PSC. [1] Moreover, in principle, the PCC in rings can induce the magnetic flux, which will modify self-consistently the PCC leading to a self-sustained magnetic Aharonov-Bohm (AB) flux in rings. [14, 15. The occurrence of the self-sustained magnetic AB flux breaks spontaneously time reversal symmetry to form the orbital magnetic phase (OMP), in which the self-sustained magnetic AB flux occurs in the ground state even turning off the external magnetic flux. 14, 15. However, for semiconductor Rashba rings both PCC and PSC can occur. 911] Whether still exist OMP in semiconductor Rashba 
rings and what is relationship between OMP and PSC? The answers of these questions will provide some helpful guideline for spintronic devices.

In this letter, we will try to answer above questions. Solving analytically the Rashba model of mesoscopic rings, we find that the electronic configuration state can be given analytically based on four kinds of electron numbers in rings. Thus,all variables, such as PCC, PSC, and the ground-state energy, can be obtained analytically so that we can give a clear understanding of whole physical story in semiconductor Rashba rings. Taking the effect of the self-inductance of the ring into account we will study the existence of self-sustained magnetic flux (SSMF) and pure PSC and analyze their relationship. We will also discuss some interesting properties of the PSC for exact one-dimensional (1D) rings.

Electrons in a two-dimensional(2D) system with a structural inversion asymmetry can be described by the so-called Rashba model, 4,5] which involves the SO interaction. For 1D mesoscopic Rashba rings under a uniform perpendicular magnetic field, the dimensionless Hamiltonian can be written as [16]

$$
\mathcal{H}=\frac{H}{E_{0}}=\left(-i \frac{\partial}{\partial \varphi}+f+\sigma_{r} \frac{\bar{\alpha}}{2}\right)^{2}-\frac{\bar{\alpha}^{2}}{4}
$$

where $E_{0}=\frac{\hbar^{2}}{2 m^{*} R^{2}}$, where $m^{*}$ is the effective mass of the electron and $R$ is the radius of ring; $f=\frac{\Phi}{\Phi_{0}}$ is the dimensionless magnetic flux, where $\Phi_{0}$ is the flux quanta; $\bar{\alpha}=\frac{\alpha}{R E_{0}}$ is the dimensionless Rashba spin-orbit coupling constant, and $\sigma_{r}=\sigma_{x} \cos \varphi+\sigma_{y} \sin \varphi$, where $\left(\sigma_{x}, \sigma_{y}\right)$ are the Pauli matrix and $\varphi$ is the angular variable in the ring. For convenience, we use the dimensionless form of all variables in the following presentation.

Solving the static Schrödinger equation with the Hamiltonian in Eq.(1) we can give the dimensionless energy spectrum,

$$
\mathcal{E}_{n, \sigma}=\left(n+f+\frac{1}{2}-\frac{\sigma}{2 \cos \theta}\right)^{2}-\frac{\tan ^{2} \theta}{4}
$$

where $\sigma= \pm 1$ for spin up and down; $n=0, \pm 1, \pm 2 \ldots$ label the electronic states, and $\tan \theta=\bar{\alpha}$. The corresponding eigenvectors can be obtained

$$
\psi_{n, \sigma}=\frac{1}{\sqrt{2 \pi}} e^{i(n+1 / 2) \varphi}\left(\begin{array}{c}
e^{-i \varphi / 2} \sin \frac{2 \theta+\pi(\sigma+1)}{4} \\
e^{i \varphi / 2} \cos \frac{2 \theta+\pi(\sigma+1)}{4}
\end{array}\right)
$$

Since the energy spectrum in Eq.(2) is a periodic function of the magnetic flux, we may consider the range of the magnetic flux within $|f|<1 / 2$. The degenerate points of the energy spectrum arise at the flux $f=-\frac{1}{2}\left(n+n^{\prime}+1-\left(\sigma+\sigma^{\prime}\right) \sqrt{1+\bar{\alpha}^{2}} / 2\right)$ for electron states $(n, \sigma)$ and $\left(n^{\prime}, \sigma^{\prime}\right)$, which divide the magnetic flux period into six ranges, $\left( \pm \frac{1}{2}, \pm f_{2}\right),\left( \pm f_{2}, \pm f_{1}\right),\left( \pm f_{1}, 0\right)$, where $f_{1}=\frac{1}{2}\left(\sqrt{1+\bar{\alpha}^{2}}-1\right)$, and $f_{2}=\frac{1}{2}\left(2-\sqrt{1+\bar{\alpha}^{2}}\right)$. For semiconductor rings with its diameter about $50 \mathrm{~nm}, \bar{\alpha} \approx 1$ and $f_{2}>f_{1}$. When $\bar{\alpha} \geq 1.118$, $f_{2} \leq f_{1}$.

At zero temperature, the configuration state of electrons is determined by electron occupation in the energy levels of the ring. For a ring with electron number $N$ and magnetic flux $f$, we find that the configuration states can be classified to four kinds of the electron number in above six ranges of the magnetic flux: case (1) $N=4 k+1$ (even number of pairs plus one extra electron), case (2) $N=4 k+2$ (odd number of pairs), case (3) $N=4 k+3$ (odd number of pairs plus one electron) and case (4) $N=4 k+4$ (even number of pairs), where $k$ is an integer. This property of electronic configuration states is similar to the non-SO coupling ring. [17 Moreover, we find that there exist a relation of the electron states with the magnetic flux $f$. For different magnetic fluxes $f$, the electron states $(n(f), \sigma(f))$ satisfy

$$
\left\{\begin{array}{l}
n(-f)+n(f)=-1 \\
\sigma(-f)+\sigma(f)=0
\end{array}\right.
$$


Table 1: The electronic state configurations in various magnetic flux ranges

\begin{tabular}{llll}
$N$ & $\left(\sum_{n \sigma} \sigma, \sum_{n \sigma} n, \sum_{n \sigma} \sigma n\right)$ & $\left(\sum_{n \sigma} \sigma, \sum_{n \sigma} n, \sum_{n \sigma} \sigma n\right)$ & $\left(\sum_{n \sigma} \sigma, \sum_{n \sigma} n, \sum_{n \sigma} \sigma n\right)$ \\
\hline $4 k+1$ & $\left(1,-\frac{3}{4}(N-1), \frac{3}{4}(N-1)\right)$ & $\left(1,-\frac{3}{4}(N-1), \frac{3}{4}(N-1)\right)$ & $\left(1,-\frac{3}{4}(N-1), \frac{3}{4}(N-1)\right)$ \\
$4 k+2$ & $\left(0,-\frac{N}{2}, \frac{N}{2}\right)$ & $\left(0,-\frac{N}{2}, \frac{N}{2}\right)$ & $(0,-N, N)$ \\
$4 k+3$ & $\left(-1,-\frac{3}{4}(N+1), \frac{3}{4}(N+1)\right)$ & $\left(-1,-\frac{3}{4}(N+1), \frac{3}{4}(N+1)\right)$ & $\left(-1,-\frac{3}{4}(N+1), \frac{3}{4}(N+1)\right)$ \\
$4 k+4$ & $\left(0,-\frac{N}{2}, N\right)$ & $\left(0,-N, \frac{N}{2}\right)$ & $\left(0,-N, \frac{N}{2}\right)$ \\
\hline$f$ & $\left(0, f_{1}\right)$ & $\left(f_{1}, f_{2}\right)$ & $\left(f_{2}, \frac{1}{2}\right)$
\end{tabular}

Notes: $f_{1}=\frac{1}{2}\left(\sqrt{1+\bar{\alpha}^{2}}-1\right)$, and $f_{2}=\frac{1}{2}\left(2-\sqrt{1+\bar{\alpha}^{2}}\right) . k$ is an integer.

This relation of electron states plays an essential role in giving analytically the symmetries of the physical quantities such as the charge current, the spin current and the ground-state energy. We can derive some rules of the occupation of electron states: (1) $\sum_{n, \sigma}(n(-f)+$ $n(f))=-N ;(2) \sum_{n, \sigma}(\sigma(-f)+\sigma(f))=0 ;(3)$ let $\lambda \equiv \sum_{n, \sigma}(\sigma(-f) n(-f)+\sigma(f) n(f))$, $\lambda=\frac{3}{2} N \pm \frac{1}{2}$ for $N=4 k+1$ and $N=4 k+3$, respectively. $\lambda=2 N$ for $N=4 k+2$ and $f_{2}<f<1 / 2$, and for $N=4 k+4$ and $0<f<f_{1} ; \lambda=N$ for $N=4 k+2$ and $0<f<f_{2}$, and for $N=4 k+4$ and $f_{1}<f<1 / 2$. We list the electron configuration states in the magnetic flux $f>0$ range in the table I.

It can be seen from the table I that the z-direction projection of the total spin of the ring in the ground state is $\left\langle S_{z}\right\rangle=\frac{\hbar}{2}\left\langle\sum_{n, \sigma} \sigma_{z}\right\rangle= \pm \frac{\hbar}{2}$ (0) for the odd-(even-)electron ring, respectively. The ground state of rings with odd-electrons is spin polarized. The spin-up and -down states depend on the different odd electrons (cases 1 and 3) and the direction of magnetic field. The ground state of rings with even-electrons is non-spin polarized. This property may be useful for spintronic devices.

The PCC and PSC can be given by $j_{c(s)}=\sum_{n, \sigma} j_{c(s), n, \sigma}$, respectively, where the sum runs over all occupied states. The PCC in each energy level can be obtained by $j_{c, n, \sigma}=-\partial \mathcal{E}_{n, \sigma} / \partial f$, and the PSC in each energy level can be calculated by $j_{s, n, \sigma}=$ $\frac{1}{2 \pi} \int_{0}^{2 \pi} 2 \operatorname{Re}\left(\psi_{n, \sigma}^{\dagger} \frac{\left\{v_{\varphi}, s_{z}\right\}}{2} \psi_{n, \sigma}\right) d \varphi$, where the velocity operator is $v_{\varphi}=2\left(-i \frac{\partial}{\partial \varphi}+f+\sigma_{r} \frac{\bar{\alpha}}{2}\right)$ [10].

At zero temperature the PCC and PSC in each energy level can be obtained

$$
\begin{aligned}
& j_{c c, n, \sigma}=-2\left(n+f+\frac{1}{2}-\frac{\sigma}{2 \cos \theta}\right) \\
& j_{s c, n, \sigma}=\left(n+f+\frac{1}{2}-\frac{\sigma}{2 \cos \theta}\right) \sigma \cos \theta
\end{aligned}
$$

Using the configuration symmetry Eq. (4) and the electron occupation rule in the table I, we can prove easily the PCC being an odd function of flux $f: j_{c}(-f)=\sum_{n, \sigma} j_{c, n, \sigma}(-f)=$ $-j_{c}(f)$, while the PSC is an even function of $f: j_{s}(-f)=\sum_{n, \sigma} j_{s, n, \sigma}(-f)=j_{s}(f)$. Therefore we may present the PCC and PSC only in half of the period $0<f<\frac{1}{2}$. Thus, we can analytically obtain the PCC,

$$
j_{c c, \text { odd }}(f)= \begin{cases}-2 N f+\frac{1}{2}\left(N-3+2 \sqrt{\bar{\alpha}^{2}+1}\right) & \text { for } N=4 k+1 \\ -2 N f+\frac{1}{2}\left(N+3-2 \sqrt{\bar{\alpha}^{2}+1}\right) & \text { for } N=4 k+3\end{cases}
$$

and

$$
j_{c c, \text { even }}(f)= \begin{cases}-N 2 f & \text { for } 0<f<f_{1,2} \\ -N(2 f-1) & \text { for } f_{1,2}<f<\frac{1}{2}\end{cases}
$$

where $f_{1,2}$ for $N=4 k+2$ and $N=4 k+4$, respectively. In Figure 1 , we plot the PCC versus the magnetic flux as an example. It can be seen that the PCC is linear with the magnetic flux and asymmetric at $f=0$. For odd-electron rings, the PCC has a jump at $f=0$. For 


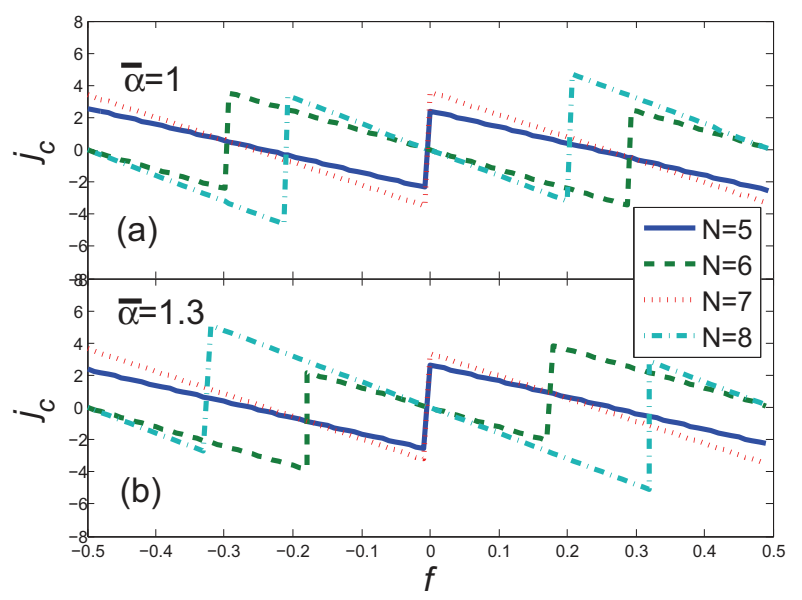

Fig. 1: The PCC versus the magnetic flux for $\bar{\alpha}=1$ in (a) and for $\bar{\alpha}=1.3$ in (b).

even-electron rings the jump points of PCC shift to $f_{2}$ and $f_{1}$ for the $(4 k+2)$ - and $(4 k+4)$ electron rings for $\bar{\alpha}=1$, respectively. For $\bar{\alpha}>1.118$, the behavior of PCC of odd-electron rings has no qualitative change, but the jumping points $f_{2}$ and $f_{1}$ of PCC exchange for the $(4 k+2)-$ and $(4 k+4)-$ number rings. A phase difference of the PCC occurs for the odd- and even- electron rings. Interestingly, there exist two symmetric non-zero magnetic fluxes corresponding to zero PCC, which is similar to the numerical result of the Rashba ring, 10] and mesoscopic hard-core boson rings with one impurity. 15. Another feature of PCC is that the PCC depends on the SO coupling strength for odd-electron rings, but is independent of the SO coupling strength for even-electron rings. For given magnetic flux $f$, when the SO coupling strength $\bar{\alpha}=\frac{1}{2} \sqrt{[(4 f-1) N \pm 3]^{2}-4}$ the PCC vanishes for oddelectron rings, but is still constant for even-electron rings. This property of PCC is similar to previous numerical results. [1] For the weak SO coupling limit the PCCs in Eqs.(7) and (87) are consistent with the PCC of the non-SO coupling case. [17.

Similarly, the PSC can be also obtained,

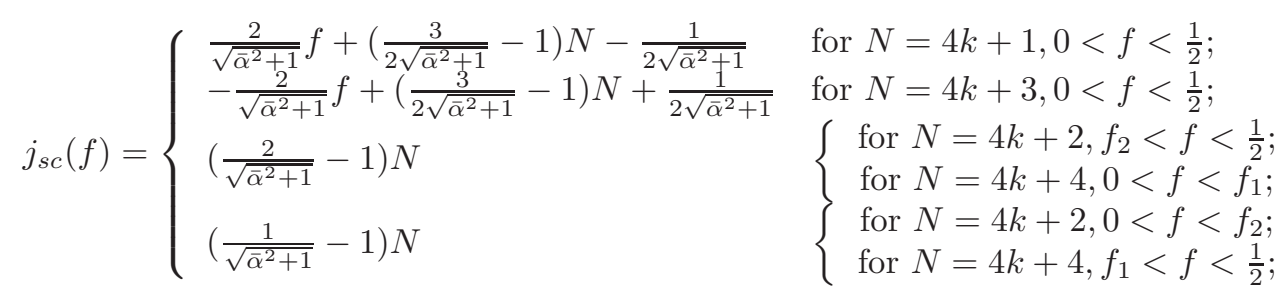

For the odd-electron rings the PSC is linear with the magnetic flux, but for the evenelectron rings the PSC is a constant. We can find that the PSC will vanish for some SO coupling strengths (see Eq.(9)), which agrees qualitatively with the numerical results. 10 The PSC versus the magnetic flux is shown in Fig. 2. We can see that the PSC is linear with the magnetic flux for odd-electron rings, but have different constants in different ranges of the magnetic flux for even-electron rings. More realistically, we may also consider 2D rings. The radial subbands will induce some additional fine structures of PCC and PSC. [10]

In principle, the $\mathrm{PCC}$ in a ring can induce a magnetic field and its corresponding magnetic flux, which interplay with the external magnetic flux to modify self-consistently the PCC. The dimensionless magnetic energy induced by the PCC can be written as $\mathcal{E}_{B}=\mu f^{2}$, where $\mu=\frac{\Phi_{0}^{2}}{2 \mathcal{L} E_{0}}$ and the self-inductance coefficient of the ring $\mathcal{L}\left[18\right.$ is $\mathcal{L}=\mu_{0} R[\ln (16 R / d)-7 / 4]$, where $d$ is the diameter of the cross section of the ring. The total energy of the whole system 


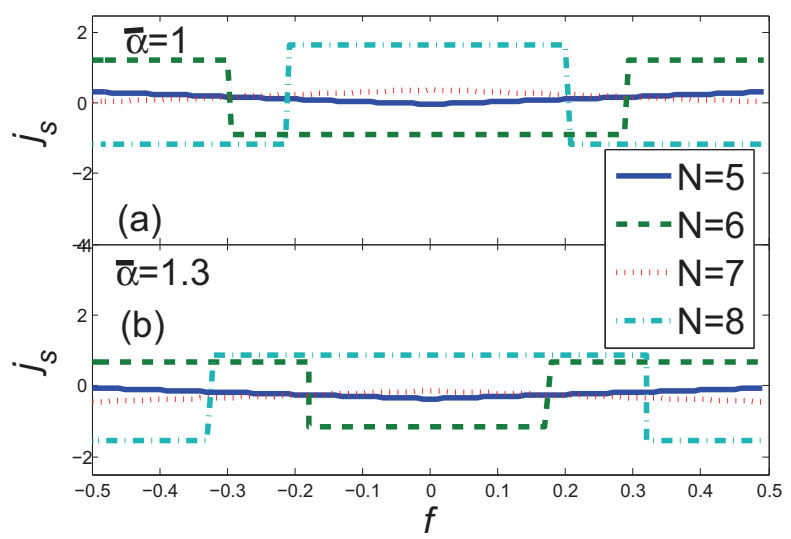

Fig. 2: The PSC versus the magnetic flux for $\bar{\alpha}=1$ in (a) and for $\bar{\alpha}=1.3$ in (b).

can be written as

$$
\mathcal{E}_{T}=\sum_{n, \sigma}\left[\left(n+f+\frac{1}{2}-\frac{\sigma}{2 \cos \theta}\right)^{2}-\frac{\tan ^{2} \theta}{4}\right]+\mu f^{2},
$$

Physically, for a given external magnetic field the stable state of the whole system should be at the minimum of the total energy $\mathcal{E}_{T}$. Solving the equation $\frac{\partial \mathcal{E}_{T}}{\partial f}=0$, we can obtain the magnetic flux of the ring in the ground state for given electron number $N$.

$$
f_{\text {odd }}^{s}= \begin{cases}\frac{1}{4(N+\mu)}\left(N-3+2 \sqrt{\bar{\alpha}^{2}+1}\right) & \text { for } N=4 k+1 \\ \frac{1}{4(N+\mu)}\left(N+3-2 \sqrt{\bar{\alpha}^{2}+1}\right) & \text { for } N=4 k+3\end{cases}
$$

and

$$
f_{\text {even }}^{s}=\frac{N}{2(N+\mu)} \text { for } N \text { even, with } f_{1,2}<f_{\text {even }}^{s}<\frac{1}{2}
$$

where $f_{1,2}$ for $N=4 k+2$ and $N=4 k+4$, respectively. Moreover, we can demonstrate $\frac{\partial^{2} \mathcal{E}_{T}}{\partial f^{2}}=2(N+\mu)$, which satisfies the condition $\left.\left(\frac{\partial^{2} \mathcal{E}_{T}}{\partial f^{2}}\right)\right|_{f=f_{s}}>0$ for $f$ in the whole period. It implies that the whole system is stable for the magnetic flux $f_{\text {odd(even) }}^{s}$ in Eqs.(11) and (12). Thus, when $f_{\text {odd(even })}^{s} \neq 0$, there exists a SSMF of the ring even though the external magnetic field is turned off after the system reaches stable states. It implies that the time reversal symmetry is broken spontaneously to form the orbital magnetic phases (OMP) like the metallic rings. 14, 15, The SSMF may be regarded as the order parameter of OMP.

The SSMF we give in Eqs.(11) and (12) depends on three parameters of the ring, the self-inductance $\mathcal{L}$ (inside $\mu$ ), the electron number $N$ and the spin-orbit coupling strength $\bar{\alpha}$. For odd-electron number rings, when $\bar{\alpha}=\sqrt{\frac{1}{4}(N \mp 3)^{2}-1}, f_{\text {odd }}^{s}=0$. This means that tuning the SO coupling strength can induce the phase transition between the orbital magnetic and non-orbital magnetic orders. This provides a possible way to tune the OMP phase transition because the SO coupling strength can be tuned by applied electric field in semiconductor heterostructures. It should be emphasized that the SSMF corresponds to a spin polarized state for odd-electron number rings, but corresponds to non-spin polarized state for even-electron number rings. This is quite different from the SSMF in metallic mesoscopic rings without SO coupling, [14, 15, in which the SSMF always corresponds to non-spin polarized state. 
Substituting the SSMF in Eqs.(11) and (12) into Eqs. (77) and (8), we can obtain the self-sustained PCC, $j_{c c}^{s}=2 \mu f_{\text {odd (even })}^{s}$. Similarly, the self-sustained PSC for odd-electron rings can be given,

$$
j_{s c}^{s}=\frac{1}{2 \sqrt{\bar{\alpha}^{2}+1}}\left(3 N-\frac{3 \pm \mu}{N+\mu}\right)+\frac{1}{N+\mu}-N,
$$

where ' + ' for $N=4 k+1$ and ' - ' for $N=4 k+3$ in the range $0<f<\frac{1}{2}$. For even-electron rings, the self-sustained PSC is still constant same to Eq. (9).

For the 1D limit $d \rightarrow 0$, the self-inductance $\mathcal{L} \rightarrow \infty$, and $\mu \rightarrow 0$, the classical magnetic field energy can be ignored. 15] The self-sustained PCC vanishes $j_{c c}^{s}=0$, but the SSMF is still finite, which is similar to the self-sustained $\mathrm{AB}$ flux in mesoscopic hard core boson ring. [15] Interestingly, the self-sustained PSC becomes,

$$
j_{s c}^{s}=\left(\frac{3}{2 \sqrt{\bar{\alpha}^{2}+1}}-1\right)\left(N-\frac{1}{N}\right)
$$

for odd- $N$. This is a self-sustained pure PSC, namely there only exists the spin current without charge current, which may be also regarded as a spontaneous time reversal symmetry breaking. This self-sustained pure PSC gives some novel properties: (1) it accompanies a nonzero magnetic flux even though it is pure PSC without PCC; (2) it is sensitive to electron number $N$ and the SO coupling strength; (3) it still survives even for weak SO couplings, but when $\bar{\alpha}=\sqrt{5} / 2$, the self-sustained pure PSC vanishes. These properties of PSC are quite different from the pure PSC predicted by Sun et.al. 11]. Theoretically, it is interesting to demonstrate the existence of the pure PSC. It has been found that the pure PSC can induce an electric field. 11,19] One has proposed a scheme to demonstrate the existence of the pure PSC by measuring this electric field. [7,20] The self-sustained pure PSC in Eq.(14) coexists with the SSMF except $\bar{\alpha}=\sqrt{5} / 2$. Thus once we can measure the magnetic field associated with the SSMF, we can demonstrate the existence of pure PSC as long as $\bar{\alpha} \neq \sqrt{5} / 2$. For the ring of $500 \mathrm{~nm}$ diameter, the magentic field of SSMF is estimated approximately as 6 Gauss.

For the Dresselhaus model, since the energy spectrum has on qualitatively difference from the Rashba, all properties we obtain from Rashba model should not have qualitatively change for the Dresselhaus model. 9]

In summary, the theoretical study of the Rashba model of mesoscopic rings reveals some quantum phenomena in semiconductor rings. The spin polarization in the ground state depends on the odd-even electron number of the ring, but the PCC and PSC can be given based on four kinds of electron numbers in the ring. The effect of the self-inductance of the ring leads to the SSMF and the self-sustained PCC and PSC, which breaks spontaneously time reversal symmetry to form OMP. The phase transition between OMP and non-OMP can be induced by tuning the $\mathrm{SO}$ coupling strength or the electron number of the ring. For exact 1D rings we find the coexistence of the pure PSC and SSMF, which may provide a theoretical scheme to measure the pure PSC.

The author gratefully acknowledges the financial supports of the projects from the National Natural Science Foundation of China (Grants No.10774194, U0634002), National Basic Research Program of China (973 Program: 2007CB935501), and Advanced Academic Research Center of Sun Yat-Sen university (06P4-3).

\section{REFERENCES}

[1] G.A. Prinz, Science, 282 (1998) 1660; S.A.Wolf, D.D. Awschalom, R.A.Buhrman,J.M. Daughton, S.von Molnar, M.L.Roukes, A.Y.Chtchelkanova, and D.M. Treger, Science, 294 (2001) 1488. 
[2] I.Zutic, J. Fabian, and S. Das Sarma, Rev. Mod. Phys., 76 (2004) 323.

[3] S. Murakami, N. Nagaosa, and S. C. Zhang, Science, 301 (2003) 1348.

[4] E. I. Rashba, Sov. Phys. Solid State, 2 (1960) 1109.

[5] J. Sinova, D. Culcer, Q. Niu, N.A. Sinitsyn, T. Jungwirth, and A.H. MacDonald, Phys. Rev. Lett., 92 (2004) 126603.

[6] S. Datta and B. Das, Appl. Phys. Lett., 56 (1990) 665; E.A. De Andrada E Silva and G.C.La Rocca, Phys. Rev. B, 59 (1999) R15583; A.A. Kiselev and K.W. Kim, Appl. Phys. Lett., 78 (2001) 775; T. Koga, J. Nitta, H. Takayanagi, and S. Datta, Phys. Rev. Lett., 88 (2001) 126601 ; J.C. Egues, G. Burkard, and D. Loss, Phys. Rev. Lett., 89 (2002) 176401; M. Governale, Phys. Rev. Lett., 89 (2002) 206802.

[7] F. Meier and D. Loss, Phys. Rev. Lett., 90 (2003) 167204; F. Schütz, M. Kollar, and P. Kopietz, Phys. Rev. Lett., 91 (2003) 017205

[8] A.F. Morpurgo, J.P. Heida, T.M. Klapwijk, and B.J. van Wees, Phys. Rev. Lett., 80 (1998) 1050; F. E. Meijer, A. F. Morpurgo, T. M. Klapwijk, T. Koga and J. Nitta, Phys. Rev. B, 69 (2004) 035308.

[9] J.S. Sheng, and Kai Chang, Phys. Rev. B, 74 (2006) 235315; R. Citro and F. Romeo, Phys. Rev. B, 75 (2007) 073306.

[10] Janine Splettstoesser, Michele Governale, and Ulrich Zülicke, Phys. Rev. B, 68 (2003) 165341.

[11] Qing-Feng Sun, X. C. Xie and Jian Wang, Phys. Rev. Lett., 98 (2007) 196801; Qing-Feng Sun, X. C. Xie and Jian Wang, Phys. Rev. B, 77 (2008) 035327; Qing-Feng Sun, Hong Guo and Jian Wang, Phys. Rev. B, 69 (2004) 054409.

[12] L.P. Levy, G. Dolan, J. Dunsmuir, and H. Bouchiat, Phys. Rev. Lett., 64 (1990) 2074.

[13] V.Chandrasekhar, R.A. Webb, M.J. Brady, M.B. Ketchen, W.J. Gallagher, and A. Kleinsasser, Phys. Rev. Lett., 67 (1991) 3578; D.Mailly, C. Chapelier, and A. Benoit, Phys. Rev. Lett., 70 (1993) 2020.

[14] D. Wohlleben, M. Esser, P.Freche, W. Zipper, and M. Szopa, Phys. Rev. Lett., 66 (1991) 3191

[15] J. X. Zhu, and Z. D. Wang, Phys. Rev. B, 51 (1995) 13813; J. X. Zhu, and Z. D. Wang, Phys. Rev. B, 52 (1995) 5275; J. X. Zhu, and Z. D. Wang, Phys. Rev. B, 53 (1996) 600; M. Y. Choi, Phys. Rev. Lett., 71 (1993) 2987.

[16] F. E. Meijer, A. F. Morpurgo, and T. M. Klapwijk, Phys. Rev. B, 66 (2002) 033107.

[17] Daniel Loss, and Paul Goldbart, Phys. Rev. B, 43 (1991) 13762.

[18] J. D. JACKson, Classical Electrodynamics (3rd ed, Wiley, NewYork) 1998, p. 234.

[19] J. E. Hirsch, Phys. Rev. B, 42 (1990) 4774; Phys. Rev. B, 60 (1999) 14787.

[20] F. Schütz, M. Kollar, and P. Kopietz, Phys. Rev. Lett., 91 (2003) 017205. 